\title{
Novel Fast Catadioptric Objective with Wide Field of View
}

\author{
Jose M. Infante Herrero ${ }^{1,3}$, Fernando Muñoz ${ }^{2}$, Pablo Benitez ${ }^{1,2}$, Juan Carlos Miñano ${ }^{1,2}$, Wang Lin ${ }^{1}$, \\ Juan Vilaplana ${ }^{2}$, Guillermo Biot ${ }^{1}$, Marta de la Fuente ${ }^{3}$. \\ ${ }^{1}$ Universidad Politécnica de Madrid, CeDInt, Campus Montegancedo, 28223 Madrid, Spain; ${ }^{2}$ Light Prescriptions Innovators-Europe, Edif. \\ CeDInt Campus Montegancedo UPM, 28223 Pozuelo, Madrid, Spain, ${ }^{3}$ Indra Sistemas SA, Joaquin Rodrigo 11, 28300 Aranjuez, Spain.
}

jose.infante@cedint.upm.es

\begin{abstract}
Using the Simultaneous Multiple Surface method in 2D (SMS2D), we present a fast catadioptric objective with a wide field of view $\left(125^{\circ} \times 96^{\circ}\right)$ designed for a microbolometer detector with $640 \times 480$ pixels and 25 microns pixel pitch.

OCIS codes: (220.3620); Optical Design and Fabrication (220.0220);
\end{abstract}

\section{Introduction}

Over the past years panoramic vision systems have been in high demand due to their use in several applications: as security cameras for buildings or perimeters, webcams for video conferences or driving cameras.

In defense applications (security or driving cameras) the infrared technology presents many more advantages than the other technologies. It is due to the fact that the thermal sensor can obtain images independently from the weather conditions or the luminosity of the scene. However these systems are still expensive compared to other sensors such as the CCDs or the intensified cameras. Depending on the application a Fish-eye [1] design can be used. Nevertheless the disadvantage of these kinds of configurations is that their range performances are limited, that is to say that they cannot be used as surveillance systems seeing as they are only capable of detecting, identifying and recognizing objects at a close distance.

The range performance of a panoramic system can be improved by means of a higher number of sensors, because the FOV (field of view) of each sensor is reduced [2]. In this article we will present a panoramic system made up of three catadioptric objectives of $125^{\circ} \times 96^{\circ}$. The objective will work between 7-14 micras. Designed for a detector of $640 \times 480$ pixels and 25 microns pixel pitch.

So as not to increase the price of the product too much, the number of optical elements that each objective can have is limited.

The design technique used is the SMS2D (Simultaneous Multiple Surfaces) method. The Simultaneous Multiple Surfaces was developed as a design method in Nonimaging Optics during the 90s. Later, the method was extended for designing Imaging Optics. The SMS design strategy is shown to perform always better than the classical design (in terms of image quality or in the number of elements used). Another advantage of this method is that the SMS method is a direct method, in other words, it is not based on multi parametric optimization. This attributes an additional interest to the SMS method since it can be used for exploring solutions where the multiparameter techniques can get lost in the multiple local minima. Note that the Classical Imaging design [3],[4],[5] is based on maximizing a certain merit function describing the image quality over the image plane. The starting point for this optimization is quite important because the algorithm progresses from an initial guess to the final solution. Since the search is local there is no guarantee that the algorithm will find the absolute maximum. For this reason the initial point must be as close as possible to the final solution.

\section{SMS approximation}

The design of one surface forming a sharp image from one point to another is a classic problem with an easy solution that is solved by means of 2nd order surfaces (parabola, ellipse or Cartesian oval) [7]. The optical surface couples one-parameter ray beam into another, and only a single ray of the beam passes through each point on the surface. Focusing on a perfect two points in two using two surfaces is a conceptually similar problem, but it is completely different in terms of implementation. In this case, for each point of the surfaces two rays always pass. Thus the design of both surfaces must be simultaneous and not sequential as in classical optics approximation, obtaining free-form surfaces instead of spherical surfaces (Fig. 1a, b). The basic SMS 
design method developed several years ago has evolved into the "image forming" version (SMS imaging), and it has been applied here as a basic tool in the realization of these designs [7]-[15].

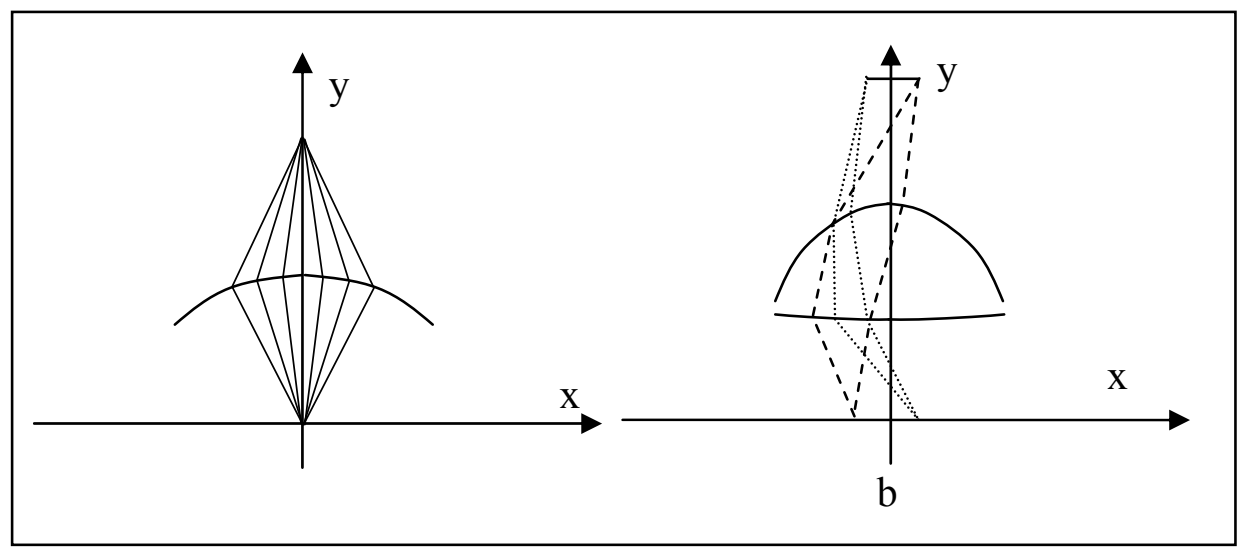

Fig.1. Two basic image-forming systems. a shows a lens with a surface that focuses a point at one point. The ease of design is due to the fact that a single beam passes through each point of the surface. $\mathbf{b}$ shows a lens that focuses on two points from two points. The design complexity is much greater because two beams pass through each point of the two surfaces.

Applying this method to this work is subject to the fulfilment of two conditions: first, it is necessary to be able to design systems that match $\mathrm{N}$ object points in $\mathrm{N}$ image points using $\mathrm{N}$ surfaces, and secondly, to be able to design systems of large angular opening. The procedure has been developed in three phases which are briefly described:

\subsection{Establishment of initial conditions of design.}

The first step is to establish the initial conditions of the problem in order to proceed to the design of free-form surfaces (in the example shown, four refracting surfaces were designed to focus on four points at infinity on a 4point receiver). Due to the nature of the SMS procedure, it is necessary to start the design with a small part of the surface in the centre, and from there to calculate all the rest (Fig. 2). We are using 2nd order surfaces without defining a parameter. Subsequently these parameters are forcing the four rays of the four input beams to focus on the four corresponding points. The solution is not simple because the law of refraction establishes four nonlinear relationships between the parameters to be determined. Using the right software, this nonlinear system of equations is solved and these small pieces of surface are taken as initial conditions of the problem. 


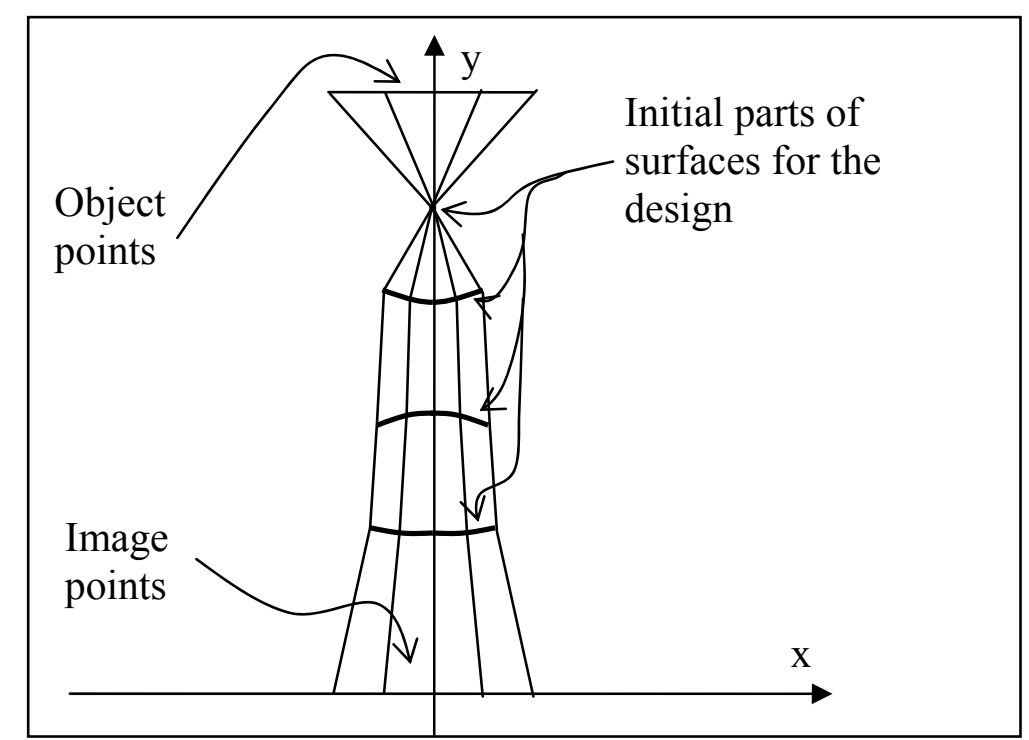

Fig.2. Initial surfaces to proceed to the design selected SMS. In this example two lenses (four surfaces) are designed to focus four points in four points. The initial three surfaces are conical that are calculated to focus the four beams.

Based on the initial conditions stated above we begin the design of the complete optical systems. The SMS procedure described in several articles [7]-[14] is used. With this method we will simultaneously find chains of surface to complete the full design. In Fig.3, an example is shown of a profile obtained. During this part of the development we have encountered many unforeseen problems related to the "instability" of the design process. While in the SMS 2D method for two surfaces the solution is always found, the method described (SMS 2D for N surfaces) the excessive curvature of the solutions near the origin results in nonfeasible solutions. This has led to the development of an algorithm for choosing the appropriate initial conditions in order to obtain practical solutions.[15][16]

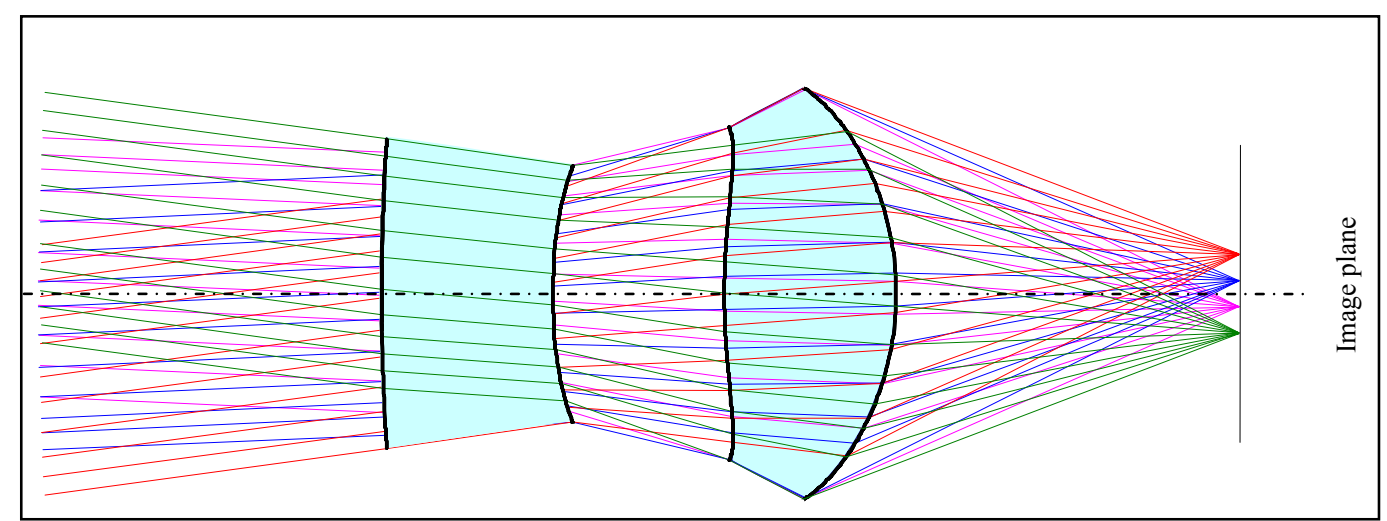

Fig 3. Example of a design obtained with the SMS 2D method to four surfaces. In this example, the object points are at infinity. [15]

\subsection{Analysis of the designs obtained.}

Finally, it is necessary to analyze the imaging quality of the result of the layouts. For this purpose we used software such as Code V. The parameter analyzed is the RMS in the focal plane, and has been found for two cases, in 2D and by rotational symmetry of the surfaces in 3D design with axial symmetry. Figure 4 shows an example of such an analysis. The results are considered reliable compared to conventional imaging, as has been published in various forums [15],[16]. 


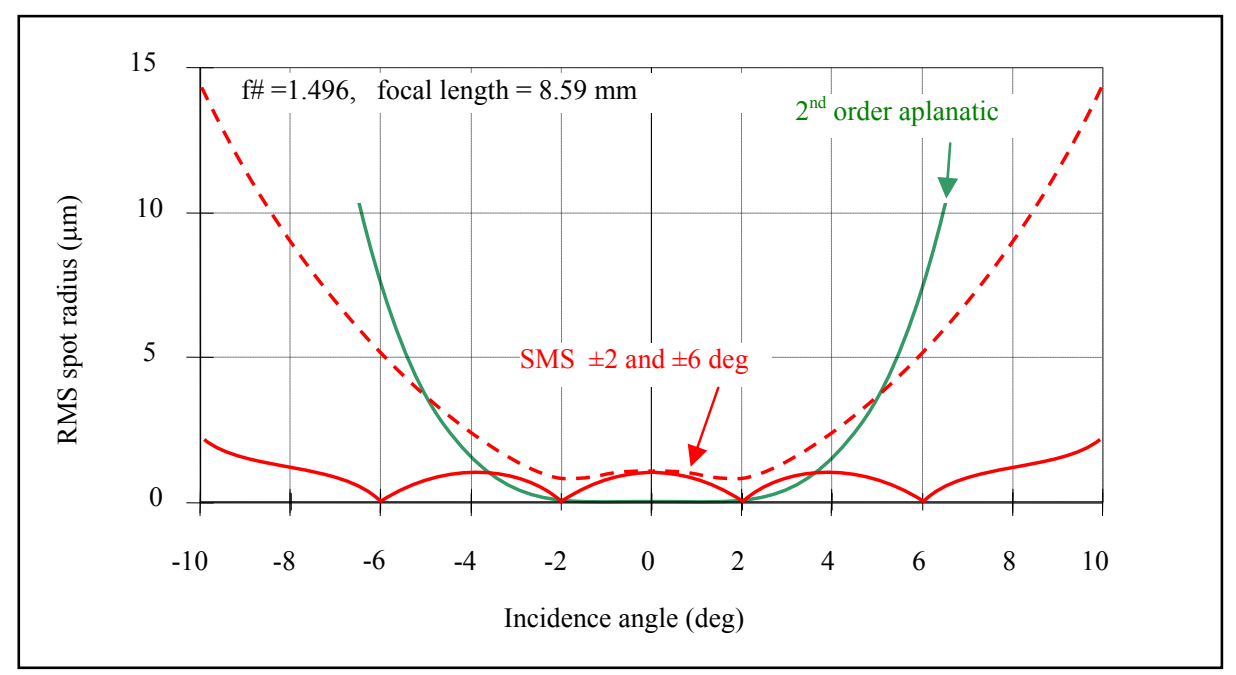

Fig 4. Comparative example of the results of a design with conventional SMS designs. The solid red line displays the RMS obtained by SMS Method for 4 surfaces. In the focal points, the RMS is zero since it was designed this way. The $2^{\text {nd }}$-order aplanatic design (green) shows a better RMS between -2 and 2 degree and because it is designed around 0 degrees. The fundamental difference is that the SMS design can increase the aperture angle to 10 degrees with a good RMS [16].

\section{Optical Lay-out}

\subsection{Approximation of the design}

Due to the fact that the objective has been designed for a microbolometers detector, the objective must be an F/1 or faster. The NETD [ref] (Noise Equivalent Temperature Difference) of the uncooled detector that we have used is $50 \mathrm{mk}$. The value of the NETD is proportional to the square of the F-Number, that value directly affects the range performance of the system.

The first decision that we made was to design a reflective system in order to avoid the chromatic aberration. The Schwarzschild configuration (figure 5) [3] partly matches the desire configuration because it is designed for faster reflective objectives.

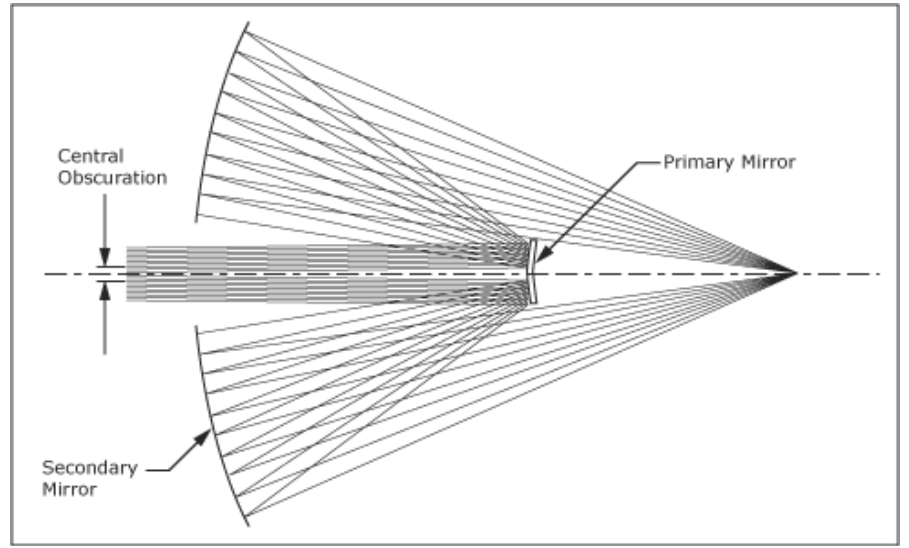

Fig 5.Schwarzschild configuration

However that configuration was designed for field of views below one degree and the primary mirror is the aperture stop of the system. In our case we are going to design the objective for the widest FOV $\left( \pm 65^{\circ} \mathrm{HFOV}\right)$, so if the primary mirror is still the aperture stop that would produce a wide hole in the secondary mirror. Another option would be to locate the aperture stop in the hole of the secondary mirror; in that case the primary 
mirror should be close to it in order to not obtain a large secondary mirror. The big problem of this solution is that in order to avoid the obscuration produces for the primary mirror, that mirror must have a wild aspheric surface. That kind of surface would produce an enormous secondary mirror for recollecting all the light and a very difficult surface for manufacturing.

It transpires that we should introduce an additional element that allows us to locate the entrance pupil in the proper place. For this reason it is necessary to put a negative meniscus in the hole of the secondary mirror. Note that in that case the primary mirror shall be the aperture stop and that we control the position of the entrance pupil controlling the power of the meniscus. If we control the position of the entrance pupil we can also control the size of the mirrors.

The meniscus should have an aspheric surface in order to control the pupil aberrations [3]. Those kinds of aberrations should be taken into account in the systems with a wide FOV.

With these premises we can use the SMS2D method in order to obtain a pre-design with three calculated surfaces that would be close enough to the optimal solution. The step from the pre-design to the final solution shall be achieved through the optimization of the surfaces given by the SMS method.

In figure 6, the steps for calculating three of the four optical surfaces of the system are shown (one of them is prescribed). We start from the initial configuration a) where $S_{1}$ and $S_{3}$ can be prescribed and $S_{2}$ is only one point. In steps b), c) and d) we calculate points of surfaces $S_{2}, S_{1}$ and $S_{3}$, using in each step respectively rays $r_{2}$, $r_{1}$ and $r_{3}$ from the corresponding design wavefronts. Steps $b, c$ and $d$ repeat themselves succesively until the curve is completed.
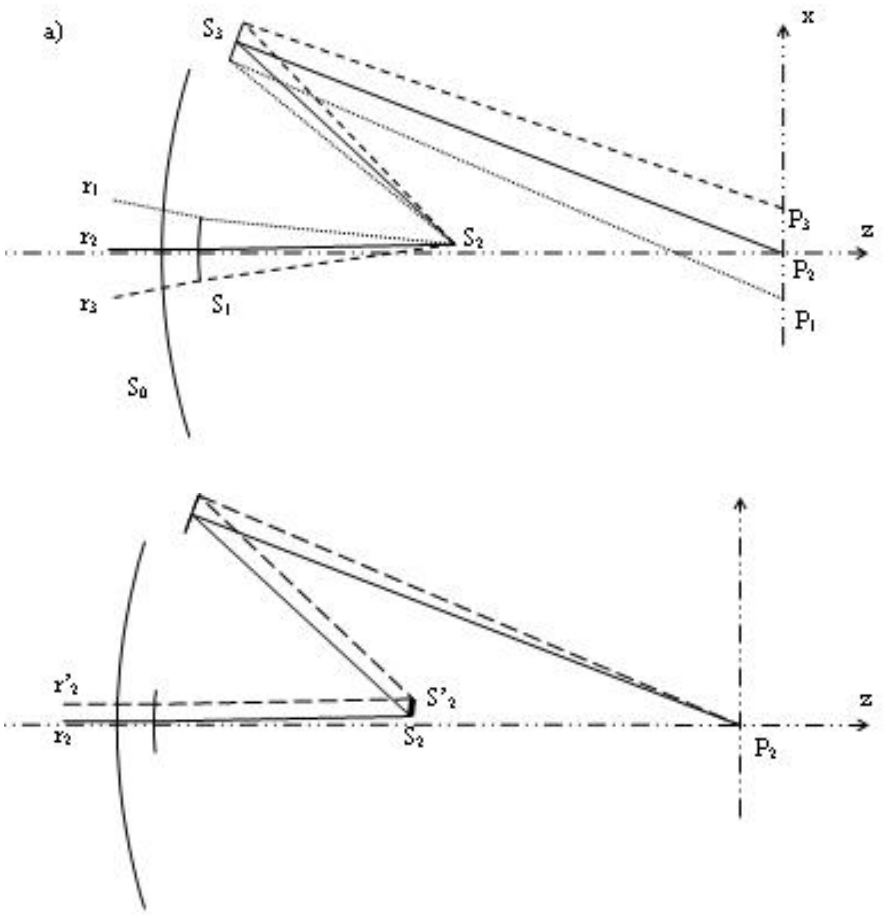

(a) 

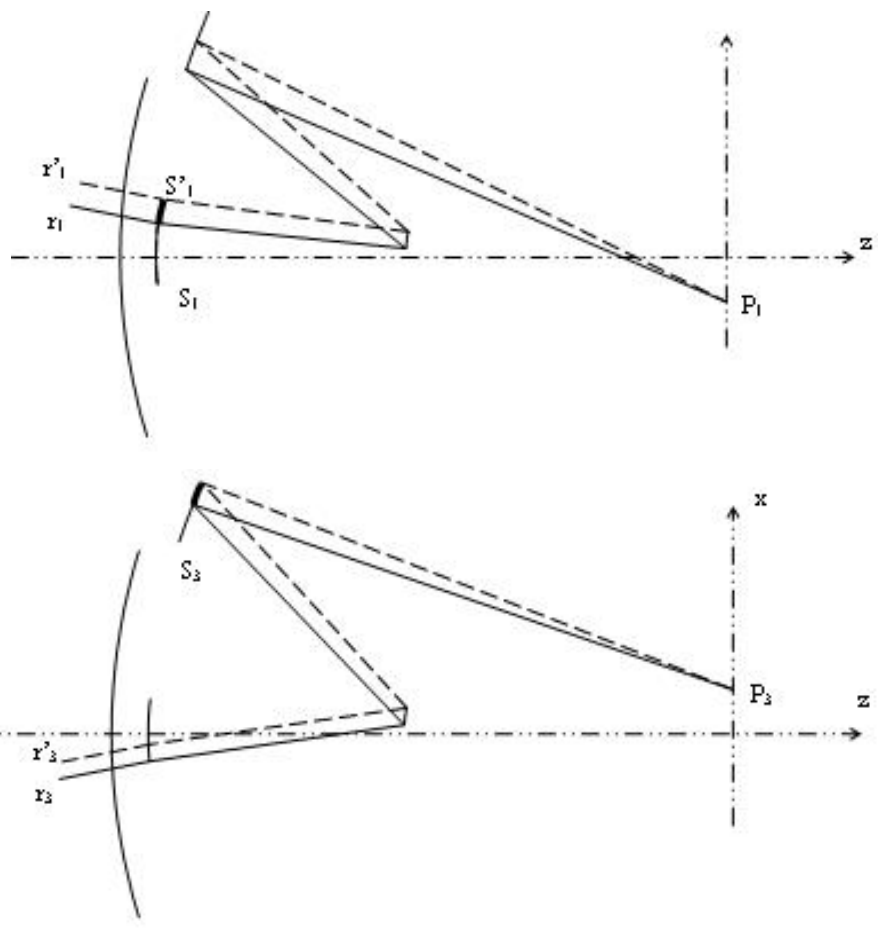

(c)

Fig 6.SMS steps for calculating surfaces $\mathrm{S}_{1}, \mathrm{~S}_{2}$ and $\mathrm{S}_{3}\left(\mathrm{~S}_{0}\right.$ is prescribed).

\subsection{Optimization Process}

One of the important parts of the design is how the merit function of the system has been defined. Our merit function has to cover all the requirements of the design that haven't been covered during the sms method and to preserve all the constants of the SMS design.

The merit function will have the effective focal length of the system, the effective F-number considering the central obstruction produced by the primary mirror, and the distortion as constants.

In order to compute the effective F-number we have to consider the amount of light at the image plane. The irradiance at the image plane is proportional to the projected solid angle (PSA) of the light cone. When we are considering systems without obstruction the projected solid angle is given by:

$$
P S A=\pi \cdot N A^{2}=\frac{\pi}{(2 \cdot \text { Fnumber })^{2}}
$$

If we have an obstruction the net PSA shall be given by:

$$
\text { EffectivePSA }=\pi \cdot\left(\mathrm{NA}_{\text {outside }}^{2}-\mathrm{NA}_{\text {inside }}^{2}\right)
$$

In the figure 7 the net projected solid angle over a draft of our system can be seen. 


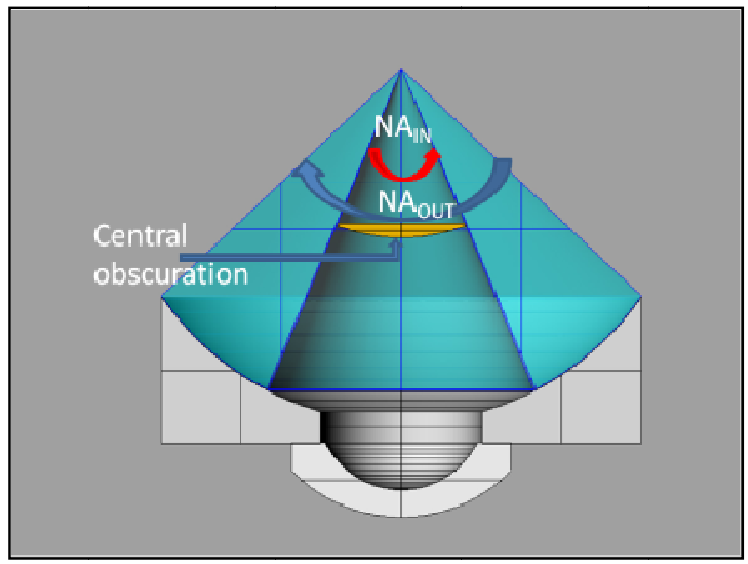

Fig 7: definition of the effective numerical aperture

If we consider (1) and (2) the effective F-number shall be given by:

$$
F / \#_{\text {eff }}=\frac{1}{2 \cdot \sqrt{N A_{\text {outside }}^{2}-N A_{\text {inside }}^{2}}}
$$

Besides those constants the weight of the different fields must be the same in order to obtain the same resolution in all the parts of the image. As the final image is going to be a composition of the three sensors the final user doesn't feel the difference when he is looking along the image.

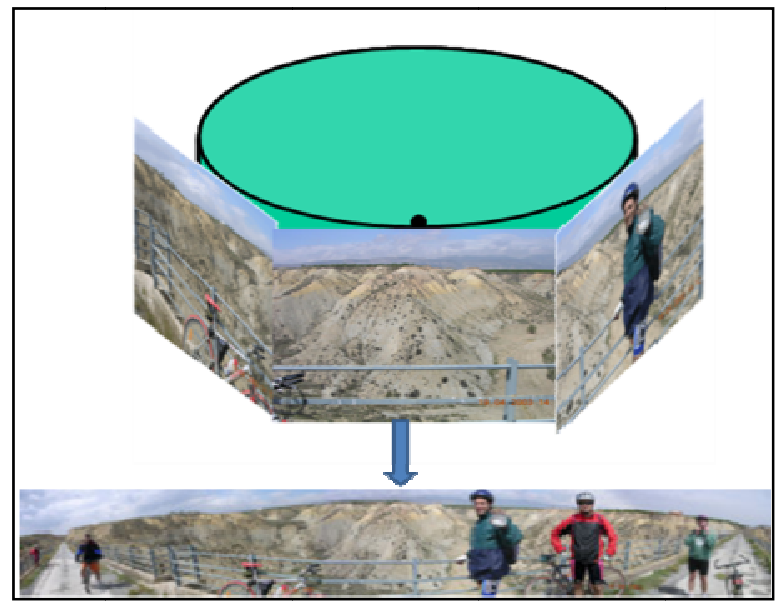

Fig 8: Composition of three images. The quality must be the same. 


\subsection{Final results}

The optical properties of the system have been summarized in the following table:

Table 1. Optical properties

\begin{tabular}{|l|l|}
\hline Waveband & $7-14$ microns \\
\hline Focal Length & $7.33 \mathrm{~mm}$ \\
\hline Effective F/\# & $\mathrm{F} / 1$ \\
\hline Working F/\# & F/0.8 \\
\hline Elements & $\begin{array}{l}\text { Front lense } \\
\text { (Germanium) Asherical/Aspherical } \\
\text { Primary mirror Aspherical } \\
\text { Secondary mirror Aspherical }\end{array}$ \\
\hline Optical Dimensions & $\begin{array}{l}142 \text { mm diameter } \\
128 \mathrm{~mm} \text { length }\end{array}$ \\
\hline
\end{tabular}

The optical system is composed of two aspheric mirrors and a negative meniscus. The negative meniscus plays a very important role in the design because it is the element that is controlling the pupil aberrations [3]. In order to reduce the cost of the lens, we have only used an aspheric surface in the lens, the other one being spherical. The primary and secondary mirrors control the aberrations in the image. In the figure the final draft of the design can be seen. Note that this configuration has enabled us to obtain a faster objective (as can be seen).

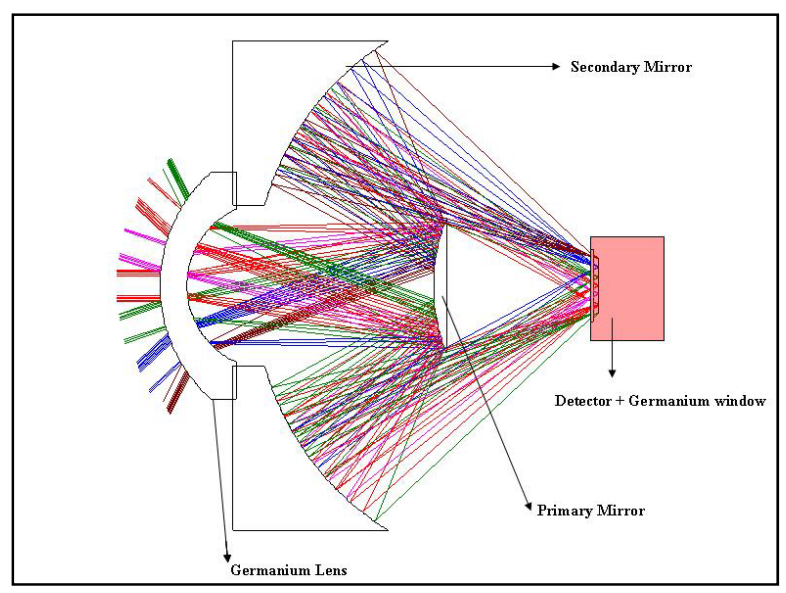

Fig 9. Final lay-out of the system

In figure 10 the image quality for all the fields can be observed. As is shown, the image quality is not exactly the same for all the fields, there is a maximum difference of $20 \%$ in the MTF. This value is acceptable because it will not create a strange effect in the image. 


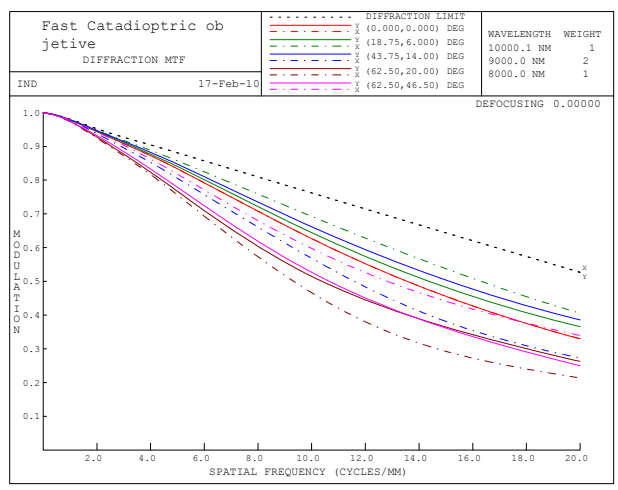

Fig 10 . MTF results of the final system

As we can see in figure 10 , the quality of the fields is very similar.We can see the average values of the MTF in Table 2. In order to attain that quality, the quality of the center of the image has been sacrificed.

\begin{tabular}{|c|c|c|}
\hline \multirow{2}{*}{ FOV } & \multicolumn{2}{|c|}{ MTF @ frequency } \\
\cline { 2 - 3 } & $10 \mathrm{cl} / \mathrm{mm}(\mathrm{T}+\mathrm{S}) / 2$ & $20 \mathrm{cl} / \mathrm{mm}(\mathrm{T}+\mathrm{S}) / 2$ \\
\hline \hline $0^{\circ}$ & $62 \%$ & $33 \%$ \\
\hline $18.75^{\circ} \times 6^{\circ}$ & $69 \%$ & $38 \%$ \\
\hline $42.75^{\circ} \times 14^{\circ}$ & $62 \%$ & $32 \%$ \\
\hline $62.50^{\circ} \times 20^{\circ}$ & $49 \%$ & $23 \%$ \\
\hline $62.50^{\circ} \times 46.5^{\circ}$ & $56 \%$ & $29 \%$ \\
\hline
\end{tabular}

An F/0.8 working F/\# involves very tight tolerances. However, the tolerances for the centering of the mirrors can be improved through using an optimization process defining different zoom positions with a slight decenter of the optical elements.

Using the movement of the detector as a compensator of tolerances, and considering all the errors produced during the manufacturing process, the maximum movement of the detector shall be of $\pm 0.24 \mathrm{~mm}$. The expected MTF considering all the possible errors in the manufacturing process and during the centering of the optical elements is presented in the table 3 .

\begin{tabular}{|c|c|c|}
\hline \multirow{2}{*}{ FOV } & \multicolumn{2}{|c|}{ MTF @ frequency } \\
\cline { 2 - 3 } & $10 \mathrm{cl} / \mathrm{mm}$ & $20 \mathrm{cl} / \mathrm{mm}$ \\
\hline \hline $0^{\circ}$ & $56 \%$ & $28 \%$ \\
\hline $18.75^{\circ} \times 6^{\circ}$ & $59 \%$ & $27 \%$ \\
\hline $42.75^{\circ} \times 14^{\circ}$ & $50 \%$ & $22 \%$ \\
\hline $62.50^{\circ} \times 20^{\circ}$ & $35 \%$ & $15 \%$ \\
\hline $62.50^{\circ} \times 46.5^{\circ}$ & $47 \%$ & $17 \%$ \\
\hline
\end{tabular}

Table 3. Expected MTF@ Frequency due to manufacturing and centering errors

\section{Optomechanical Design.}

Such a large field of view of the system requires a large opening along the optical path. A baffle is included to avoid rays from the scene going straight to the detector, without going through the optical path. The athermalization requires that the detector be moved by means of a motor along the optical axis. 

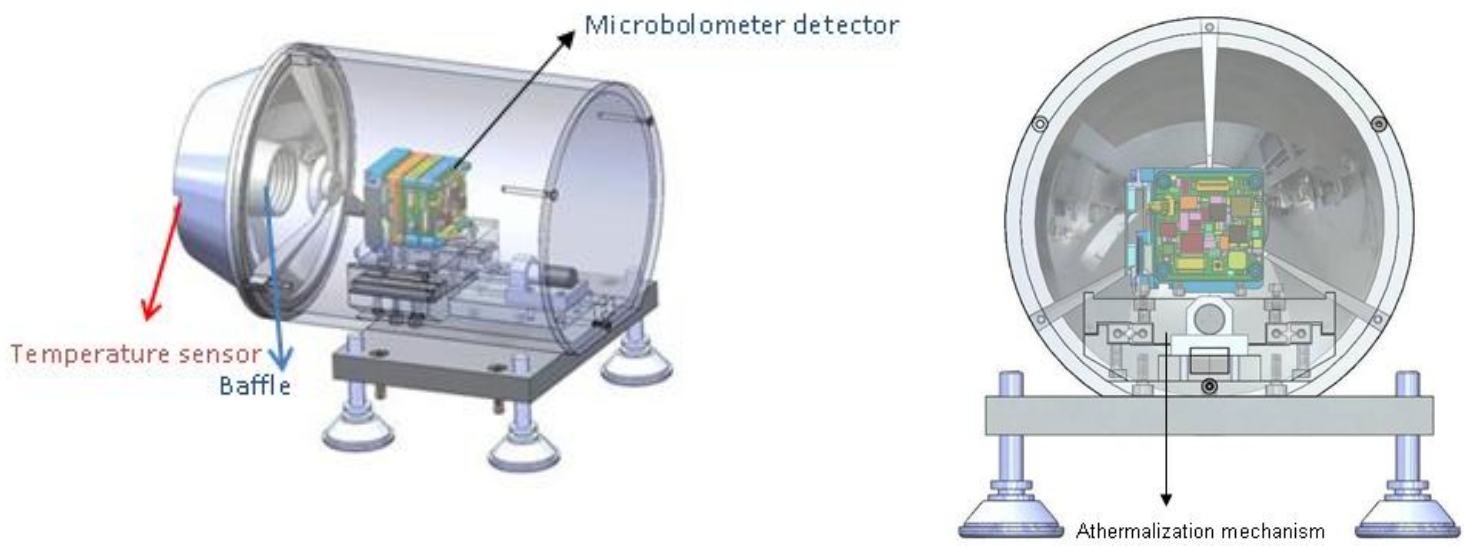

Fig 11. Opt mechanical layout

Due to the effect of the temperature in the Germaniun lens a temperature sensor has been located in the mount of the lenses in order to control the athermalization. Note that the effective focal length of the whole system has a strong dependence with this lens so a variation in the temperature of the lenses produces a variation in the focus point. As there is a linear relation between the temperature of the lenses and the position of the focus point, an algorithm has been implemented in order to move the position of the sensor depending on the temperature.

\section{Conclusions}

We have shown a new strategy for designing optical systems where we use the SMS method as the starting point for a later optimization. The SMS method ensures that the input and output bundles become fully coupled by the optical system. That fact allows us not only to obtain a good starting point, but also the design can have the optimum number of optical elements. It is very important from a certain point of view because we can reduce the cost of the optics as well. The later optimization process is also improved because it can be fulfilled faster and more efficiently due to the fact that the starting point is very close to the final solution.

Using that philosophy, we have designed a catadioptric objetive with a very wide field of view. The manufacturing of the camera is currently ongoing.

\section{ACKNOWLEDGEMENT}

The LPI's devices presented in this paper are protected under US and International patents pending by LPI LLC, 2400Lincoln Avenue, Altadena, CA 91001 USA http://www.lpi-llc.com/. The authors thank Spanish Ministries MITYC (Osv:TSI-02303-2008-52), the Madrid Regional Government (Abl: PIE/466/2009, F3: PIE/469/2009) and UPM (Q090935C-019) for their partial support. The authors also thank ORA customer service for all the help towards developing some of the macros for the optimization process. In particular we would like to express our gratitude to John F. Isenberg for his help.

\section{REFERENCES}

[1]K.Miyamoto "Fish-eye lens", JOSA,Vol. 54,1964

[2]Gerald C. Holst, Electro-Optical System Performance (JDC publishing,2008)

[3] R. Kingslake, Lens Design Fundamentals (Academic, New York, 1978).

[4] W. J. Smith, Modern Optical Engineering, 3rd ed. (McGraw-Hill, 2000).

[5] R. E. Fisher and B. Tadic-Galeb, Optical System Design (McGraw-Hill, 2000).

[6]Arnold Daniels,Field Guide to Infrared Systems (SPIE PRESS 2007) 
[7] Chaves, J., [Introduction to Nonimaging Optics], CRC Press, (2008).

[8] Winston, R., Miñano, J.C., Benítez, P., [Nonimaging Optics], Academic Press, New York, (2005).

[9] Miñano, J.C., Benítez, P., Chaves, J., Falicoff, W., Parkyn, W., "Etendue-conserving illumination-optics for backlights and frontlights", US patent application

[10] Miñano, J.C., Benítez, P., Chaves, J., Hernandez, M., Dross, O. and Santamaria, A., "High-efficiency LED backlight optics designed with the flow-line method", Proc. SPIE 5942, (2005).

[11] Chaves, J., Falicoff, W., Miñano, J.C., Benítez, P., Parkyn, W., Alvarez, R., Dross, O., “Optical manifold for light-emitting diodes", United States Patent 7380962

[12] Chaves, J., Falicoff, W., Dross, O., Miñano, J.C., Benítez, P., Parkyn, W., "Combination of light sources and light distribution using manifold optics", Proc. SPIE 6338, (2006).

[13] Cvetkovic, A., Dross, O., Chaves, J., Benítez, P., Miñano, J.C., and Mohedano, R., "Etendue-preserving mixingand projection optics for high-luminance LEDs, applied to automotive headlamps", Optics Express, 14 (26),13014-13020 (2006).

[14] Dross, O., Miñano, J.C., Benítez, P., Cvetkovic, A., Chaves, J., "Non-imaging optics combine LEDs into one bright source", SPIE Newsroom, http://newsroom.spie.org/x3596.xml (2006).

[15] Dross, O., Cvetkovic, A., Chaves, J., Benítez, P., Miñano, J.C., "LED Headlight Architecture that creates a High Quality Beam Pattern independent of LED Shortcomings", Proc. SPIE 5942, 126-135 (2005).

[16] Miñano, J.C., González, J.C., "New method of design of nonimaging concentrators", Appl. Opt. 31, 30513060 (1992).

[17] Miñano, J.C., Benítez, P., Wang-Lin,, Muñoz, F., Infante, J., Santamaría, A., "Overview of the SMS design method applied to imaging optics", Proc. SPIE 7429, 74290C (2009).

[18] Miñano, J.C., Benítez, P., Wang-Lin,, Infante, J., Muñoz, F., Santamaría, A., "An application of the SMS method for imaging designs," Opt. Express 17, 24036-24044 (2009).

[19] Fernando Muñoz Doctoral Disertation "Sistemas ópticos avanzados de gran compactibiliad con aplicaciones en formación de imagen y en iluminación” UPM, Madrid 2004 